\title{
A boundary element method for the numerical solution of a class of elliptic boundary value problems for anisotropic inhomogeneous media
}

\author{
Moh. Ivan Azis* David L.Clements ${ }^{\dagger}$ \\ Wono S. Budhi
}

(Received 1 June 2001; revised 30 October 2002)

\begin{abstract}
A boundary element method is obtained for a class of two dimensional elliptic boundary value problems for inhomogeneous media. The method can be applied to variety of problems in such areas as antiplane strain in elastostatics, plane thermostatic problems for inhomogeneous anisotropic materials and flow through porous media.
\end{abstract}

*Department of Applied Mathematics, University of Adelaide, Australia. mailto:mazis@maths.adelaide.edu.au

$\dagger$ as above, mailto: dclement@maths . adelaide.edu.au

$\ddagger$ Department of Mathematics, Institut Teknologi Bandung, InDonEsIA, mailto:wono@dns.math.itb.ac.id

${ }^{0}$ See http: //anziamj.austms.org.au/V44/CTAC2001/Azi2 for this article, (C) Austral. Mathematical Soc. 2003. Published 1 April 2003. ISSN 1446-8735 


\section{Contents}

1 Introduction $\quad \mathbf{C 8 0}$

2 The boundary value problem $\quad$ C81

3 A perturbation method $\quad$ C82

4 Numerical results $\quad$ C87

4.1 Problem $1 \ldots \ldots \ldots \ldots \ldots$ C . . . . . . . . . 87

4.2 Problem $2 \ldots \ldots \ldots \ldots \ldots \ldots$ C . . . . . . . . . . .

5 Summary

C93

References

C94

\section{Introduction}

Whereas the boundary element method provides an effective numerical procedure for the solution of elliptic boundary value problems for homogeneous media the same is not generally true for inhomogeneous media. In recent years some progress has been made in using the method to solve problems for inhomogeneous isotropic materials (see for example Clements [4], Cheng [2, 3], Rangogni [9], Shaw [10], Gipson, Ortiz and Shaw [8] and Ang, Kusuma and Clements [1]). In the case of anisotropic inhomogeneous media there are few published works. An elliptic equation which is relevant for a certain class of problems for anisotropic inhomogeneous media has been considered by Clements and Rogers [7].

The present paper is concerned with obtaining a boundary integral equation for the solution of boundary value problems governed 
by equations of the form

$$
\frac{\partial}{\partial x_{i}}\left[\lambda_{i j}\left(x_{1}, x_{2}\right) \frac{\partial \phi\left(x_{1}, x_{2}\right)}{\partial x_{j}}\right]=0
$$

where the repeated summation convention (summing from 1 to 2) is employed. Equations of this type govern the behaviour of a wide class of boundary value problems for both isotropic and anisotropic inhomogeneous media. Antiplane strain in elastostatics, plane thermostatics for anisotropic inhomogeneous materials and flow through porous media are three areas for which the governing equation is of the type (1).

A particular technique for solving a restricted class of equations of the type (1) has been obtained by Clements and Budhi [6]. Here a further technique will be considered for obtaining boundary integral equations for the solution of (1) for a more general class of equations of the type (1).

Some numerical examples are considered to illustrate the application of the boundary integral equations.

The analysis of the paper is purely formal; the main aim being to construct an effective boundary element method for classes of equations which fall within the type (1).

\section{The boundary value problem}

A solution to (1) is sought which is valid in a region $\Omega$ in $R^{2}$ with boundary $\partial \Omega$ which consists of a finite number of piecewise smooth closed curves. On $\partial \Omega_{1}$ the dependent variable $\phi(\mathbf{x})$ (where $\left.\mathbf{x}=\left(x_{1}, x_{2}\right)\right)$ is specified whereas on $\partial \Omega_{2}, P=\lambda_{i j}\left(\partial \phi / \partial x_{j}\right) n_{i}$ is 
specified where $\partial \Omega=\partial \Omega_{1} \cup \partial \Omega_{2}$ and $\mathbf{n}=\left(n_{1}, n_{2}\right)$ denotes the outward pointing normal to $\partial \Omega$.

For all points in $\Omega$ the matrix of coefficients $\left[\lambda_{i j}(\mathbf{x})\right]$ is a real symmetric positive definite matrix so that throughout $\Omega$ equation (1) is a second order elliptic partial differential equation. Further the coefficients $\lambda_{i j}(\mathbf{x})$ are required to be twice differentiable functions of the two independent variables.

The method of solution will be to obtain a boundary integral equation from which numerical values of the dependent variable $\phi$ and $P$ may be obtained for all points in $\Omega$.

The analysis of this paper is especially relevant to an anisotropic medium but it equally applies to the isotropic case. For isotropy the coefficients in (1) take the form $\lambda_{11}=\lambda_{22}$ and $\lambda_{12}=0$; use of these relations in the following analysis immediately yields the corresponding results for an isotropic material.

\section{A perturbation method}

Consider the case when the coefficients $\lambda_{i j}(\mathbf{x})$ in equation (1) may be written in the form

$$
\lambda_{i j}(\mathbf{x})=\lambda_{i j}^{(0)} g(\mathbf{x})+\epsilon \lambda_{i j}^{(1)}(\mathbf{x}),
$$

where the constant coefficients $\lambda_{i j}^{(0)} \geq 0$ for $i, j=1,2, \lambda_{11}^{(0)} \lambda_{22}^{(0)}-$ $\lambda_{12}^{(0) 2}>0, g(\mathbf{x})>0, \lambda_{i j}^{(1)}(\mathbf{x})$ and $g(\mathbf{x})$ are twice differentiable functions and $\epsilon$ is a small parameter. Substitution of (2) into (1) provides

$$
\lambda_{i j}^{(0)} \frac{\partial}{\partial x_{i}}\left[g \frac{\partial \phi}{\partial x_{j}}\right]=-\epsilon \frac{\partial}{\partial x_{i}}\left[\lambda_{i j}^{(1)} \frac{\partial \phi}{\partial x_{j}}\right] .
$$


Let

$$
\psi(\mathbf{x})=g^{1 / 2}(\mathbf{x}) \phi(\mathbf{x}),
$$

where $g^{1 / 2}(\mathbf{x})$ denotes the positive square root of $g(\mathbf{x})$. This transformation permits the left hand side of (3) to be written in the form

$$
\lambda_{i j}^{(0)} \frac{\partial}{\partial x_{i}}\left[g \frac{\partial}{\partial x_{j}}\left(g^{-1 / 2} \psi\right)\right]=\lambda_{i j}^{(0)} \frac{\partial^{2} \psi}{\partial x_{i} \partial x_{j}}-g^{-1 / 2} \lambda_{i j}^{(0)} \frac{\partial^{2} g^{1 / 2}}{\partial x_{i} \partial x_{j}} \psi .
$$

Hence substitution of (4) into (3) provides

$$
\lambda_{i j}^{(0)} \frac{\partial^{2} \psi}{\partial x_{i} \partial x_{j}}-g^{-1 / 2} \lambda_{i j}^{(0)} \frac{\partial^{2} g^{1 / 2}}{\partial x_{i} \partial x_{j}} \psi=-\epsilon \frac{\partial}{\partial x_{i}}\left[\lambda_{i j}^{(1)} \frac{\partial}{\partial x_{j}}\left(g^{-1 / 2} \psi\right)\right] .
$$

If $g(\mathbf{x})$ is restricted to satisfy the equation

$$
\lambda_{i j}^{(0)} \frac{\partial^{2} g^{1 / 2}}{\partial x_{i} \partial x_{j}}=0
$$

then equation (5) reduces to

$$
\lambda_{i j}^{(0)} \frac{\partial^{2} \psi}{\partial x_{i} \partial x_{j}}=-\epsilon \frac{\partial}{\partial x_{i}}\left[\lambda_{i j}^{(1)} \frac{\partial}{\partial x_{j}}\left(g^{-1 / 2} \psi\right)\right] .
$$

A simplification on the right hand side of (7) gives

$$
\lambda_{i j}^{(0)} \frac{\partial^{2} \psi}{\partial x_{i} \partial x_{j}}=-\epsilon\left[\frac{\partial A_{i}}{\partial x_{i}} \psi+\left(A_{j}+\frac{\partial B_{i j}}{\partial x_{i}}\right) \frac{\partial \psi}{\partial x_{j}}+B_{i j} \frac{\partial^{2} \psi}{\partial x_{i} \partial x_{j}}\right],
$$

where

$$
A_{i}(\mathbf{x})=\lambda_{i j}^{(1)} \frac{\partial g^{-1 / 2}}{\partial x_{j}}, \quad B_{i j}(\mathbf{x})=\lambda_{i j}^{(1)} g^{-1 / 2} .
$$

A solution to equation (8) is sought in the form

$$
\psi(\mathbf{x})=\sum_{r=0}^{\infty} \epsilon^{r} \psi^{(r)}(\mathbf{x}) .
$$


Substitution of (9) into (8) and equating coefficients of powers of $\epsilon$ yields

$$
\lambda_{i j}^{(0)} \frac{\partial^{2} \psi^{(r)}}{\partial x_{i} \partial x_{j}}=h^{(r)} \quad \text { for } r=0,1, \ldots,
$$

where

$$
\begin{aligned}
h^{(0)}(\mathbf{x})= & 0, \\
h^{(r)}(\mathbf{x})= & -g^{-1 / 2}\left[\frac{\partial A_{i}}{\partial x_{i}} \psi^{(r-1)}+\left(A_{j}+\frac{\partial B_{i j}}{\partial x_{i}}\right) \frac{\partial \psi^{(r-1)}}{\partial x_{j}}\right. \\
& \left.\quad+B_{i j} \frac{\partial^{2} \psi^{(r-1)}}{\partial x_{i} \partial x_{j}}\right],
\end{aligned}
$$

for $r=1,2, \ldots$.

The integral equation for (10), for $r=0,1, \ldots$, is [5]

$$
\begin{aligned}
& \eta \psi^{(r)}\left(\mathbf{x}_{0}\right)=\int_{\Omega} h^{(r)}(\mathbf{x}) \Phi\left(\mathbf{x}, \mathbf{x}_{0}\right) d S(\mathbf{x}) \\
& \quad+\int_{\partial \Omega}\left[\Gamma\left(\mathbf{x}, \mathbf{x}_{0}\right) \psi^{(r)}(\mathbf{x})-\Phi\left(\mathbf{x}, \mathbf{x}_{0}\right) P^{\left[\psi^{(r)}\right]}(\mathbf{x})\right] d s(\mathbf{x}),
\end{aligned}
$$

where $P^{\left[\psi^{(r)}\right]}=\lambda_{i j}^{(0)}\left(\partial \psi^{(r)} / \partial x_{j}\right) n_{i}, \mathbf{x}_{\mathbf{0}}=(a, b), \eta=1$ if $(a, b) \in \Omega$ and $\eta=\frac{1}{2}$ if $(a, b) \in \partial \Omega$ and $\partial \Omega$ has a continuously turning tangent at $(a, b)$. Also

$$
\begin{aligned}
\Phi & =\frac{-\lambda_{11}^{(0)}}{2 \lambda_{22}^{(0)} \pi i(\tau-\bar{\tau})}\{\log (z-c)+\log (\bar{z}-\bar{c})\} \\
\Gamma & =\frac{\mathcal{M}}{z-c}+\frac{\overline{\mathcal{M}}}{\bar{z}-\bar{c}},
\end{aligned}
$$

with

$$
\mathcal{M}=\frac{-\lambda_{11}^{(0)}}{2 \lambda_{22}^{(0)} \pi i(\tau-\bar{\tau})}\left\{\lambda_{11}^{(0)} n_{1}+\lambda_{12}^{(0)} n_{2}+\left(\lambda_{12}^{(0)} n_{1}+\lambda_{22}^{(0)} n_{2}\right) \tau\right\},
$$


where $z=x_{1}+\tau x_{2}, c=a+\tau b$ and $\tau$ is the root with positive imaginary part of the quadratic

$$
\lambda_{11}^{(0)}+2 \lambda_{12}^{(0)} \tau+\lambda_{22}^{(0)} \tau^{2}=0
$$

and the bar denotes the complex conjugate.

From (4) and (9) $\phi$ is given by

$$
\phi(\mathbf{x})=\sum_{r=0}^{\infty} \epsilon^{r} \phi^{(r)}(\mathbf{x}),
$$

where use of (4) in (16) provides

$$
\psi^{(r)}=g^{1 / 2} \phi^{(r)} \quad \text { for } r=0,1, \ldots .
$$

Also

$$
P^{\left[\psi^{(r)}\right]}=g^{1 / 2} P^{(r)}+\phi^{(r)} P^{[g]} \quad \text { for } r=0,1, \ldots,
$$

where

$$
\begin{aligned}
P^{(r)}(\mathbf{x}) & =\lambda_{i j}^{(0)} \frac{\partial \phi^{(r)}}{\partial x_{j}} n_{i} \text { for } r=0,1, \ldots \\
P^{[g]}(\mathbf{x}) & =\lambda_{i j}^{(0)} \frac{\partial g^{1 / 2}}{\partial x_{j}} n_{i} .
\end{aligned}
$$

Thus the integral equation (12) may be written in the form

$$
\begin{aligned}
& \eta g^{1 / 2}\left(\mathbf{x}_{0}\right) \phi^{(r)}\left(\mathbf{x}_{0}\right) \\
& =\int_{\partial \Omega}\left\{\left[g^{1 / 2}(\mathbf{x}) \Gamma\left(\mathbf{x}, \mathbf{x}_{0}\right)-P^{[g]}(\mathbf{x}) \Phi\left(\mathbf{x}, \mathbf{x}_{0}\right)\right] \phi^{(r)}(\mathbf{x})\right. \\
& \left.\quad-\left[g^{-1 / 2}(\mathbf{x}) \Phi\left(\mathbf{x}, \mathbf{x}_{0}\right)\right] P^{(r)}(\mathbf{x})\right\} d s(\mathbf{x}) \\
& +\int_{\Omega} h^{(r)}(\mathbf{x}) \Phi\left(\mathbf{x}, \mathbf{x}_{0}\right) d S(\mathbf{x}) .
\end{aligned}
$$


Also, the function $h^{(r)}$ in (11), for $r=1,2, \ldots$, may be written as

$$
\begin{aligned}
h^{(r)}= & -g^{-1 / 2}\left\{\left[\frac{\partial}{\partial x_{i}}\left(g^{1 / 2} A_{i}\right)+\frac{\partial B_{i j}}{\partial x_{i}} \frac{\partial g^{1 / 2}}{\partial x_{j}}\right] \phi^{(r-1)}\right. \\
& +\left[g^{1 / 2} A_{i}+\frac{\partial}{\partial x_{j}}\left(g^{1 / 2} B_{i j}\right)+B_{i j} \frac{\partial g^{1 / 2}}{\partial x_{j}}\right] \frac{\partial \phi^{(r-1)}}{\partial x_{i}} \\
& \left.+\left(g^{1 / 2} B_{i j}\right) \frac{\partial^{2} \phi^{(r-1)}}{\partial x_{j} \partial x_{i}}\right\} .
\end{aligned}
$$

Now, the corresponding value of $P$ is given by

$$
P=\lambda_{i j} \frac{\partial \phi}{\partial x_{j}} n_{i}=g P^{(0)}+\sum_{r=1}^{\infty} \epsilon^{r}\left[g P^{(r)}+G^{(r)}\right],
$$

where

$$
G^{(r)}(\mathbf{x})=\lambda_{i j}^{(1)} \frac{\partial \phi^{(r-1)}}{\partial x_{i}} n_{j} \quad \text { for } r=1,2, \ldots
$$

To satisfy the boundary conditions in Section 2 it is required that $\phi^{(0)}=\phi$ on $\partial \Omega_{1}$ where $\phi$ takes on its specified value on $\partial \Omega_{1}$. Also it is required that on $\partial \Omega_{2} P^{(0)}=g^{-1} P$ where $P$ takes on its specified value on $\partial \Omega_{2}$. It then follows from (16) and (20) that for $r=1,2, \ldots \phi^{(r)}=0$ on $\partial \Omega_{1}$ and $P^{(r)}=-g^{-1} G^{(r)}$ on $\partial \Omega_{2}$.

The integral equation (18) may now be used to find the numerical values of the unknowns on the boundary $\partial \Omega$ and the numerical values of $\phi^{(r)}$ and derivatives in the domain $\Omega$ for $r=0,1, \ldots$ At each stage in using (18) to determine $\phi^{(r)}$, the $G^{(r)}$ occuring in the boundary condition $P^{(r)}=-g^{-1} G^{(r)}$ on $\partial \Omega_{2}$ may be obtained from (21) which is evaluated from the previous iteration. Equations (16) and (20) then provide the values of $\phi$ in the domain $\Omega$ and $P$ on the boundary $\partial \Omega$. 


\section{Numerical results}

In this section various particular boundary value problems are solved numerically by employing the integral equations obtained in the previous section. In implementing the method to obtain numerical results standard boundary element procedures are employed [5, e.g.]. For all the problems considered the domain $\Omega$ is taken to be a square of side $l$ and each side of the boundary $\partial \Omega$ is divided into $N$ segments of equal length. Simpson's rule is applied to evaluate the line integrals on each segment. For the evaluation of domain integrals the domain $\Omega$ is divided into $N^{2}$ equal rectangles and the integrand is assumed to be constant over each rectangle.

\subsection{Problem 1}

Consider the problem of determining the temperature $T$ throughout the square region in Figure 1 with the boundary conditions (as shown in Figure 1)

$$
\begin{aligned}
& \bar{T}=0.181818 \quad \text { on } \mathrm{BC}, \\
& \bar{T}=0.1 \quad \text { on } \mathrm{AD}, \\
& \bar{P}=0 \quad \text { on } \mathrm{AB} \text { and } \mathrm{CD},
\end{aligned}
$$

where $\bar{T}=T / T^{*}$ with $T^{*}$ a reference temperature. Also $\bar{x}_{i}=x_{i} / l$ for $i=1,2, \bar{P}=P / P^{*}$ with $P^{*}=\lambda T^{*} / l$ where $\lambda$ and $l$ denote, respectively, a reference thermal conduction coefficient and reference length.

The thermal conduction coefficients $\lambda_{i j}$ for the material occupying the region in Figure 1 increase in magnitude from $\bar{x}_{1}=0$ to $\bar{x}_{1}=1$ according to the relationship

$$
\bar{\lambda}_{i j}=\left\{1+0.1\left(2 \bar{x}_{1}+0.1 \bar{x}_{1}^{2}\right)\right\}\left[\begin{array}{ll}
1 & 0 \\
0 & 2
\end{array}\right]
$$




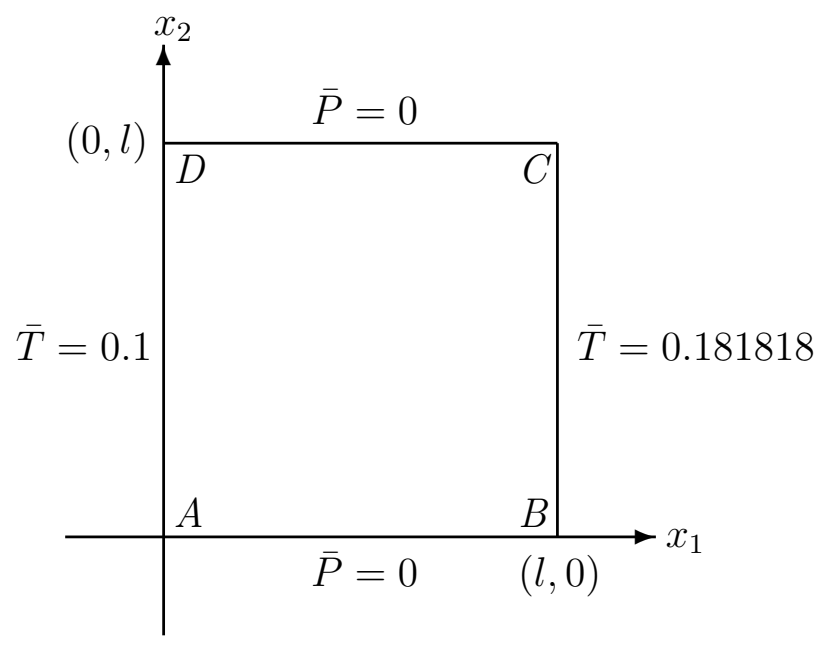

Figure 1: The geometry for Problem 1

where $\bar{\lambda}_{i j}=\lambda_{i j} / \lambda$.

This boundary value problem admits the analytic solution

$$
\bar{T}=\frac{0.1+0.1 \bar{x}_{1}}{1+0.1 \bar{x}_{1}} .
$$

The problem is now solved using the numerical technique of Section 3. In applying this technique there are a number of possibilities in the choice of $g(\mathbf{x})$ and $\lambda_{i j}^{(1)}(\mathbf{x})$ in order to provide the coefficients (22) from the form given in equation (2). Two possible forms for $g(\mathbf{x})$ and $\lambda_{i j}^{(1)}(\mathbf{x})$ are considered here.

1. Here $g(\mathbf{x})$ and $\lambda_{i j}^{(1)}(\mathbf{x})$ may be taken in the form

$$
g(\mathbf{x})=1, \quad \epsilon=0.1
$$


TABLE 1: Numerical and analytic solutions for Problem 1

$\begin{array}{lllll}\text { Position } & \bar{T} \text { BEM } & \bar{T} \text { BEM } & \bar{T} \text { BEM } & \bar{T}\end{array}$

\begin{tabular}{ccccc}
$\left(\bar{x}_{1}, \bar{x}_{2}\right)$ & 8 segments & 32 segments & 64 segments & Analytic \\
\hline$(0.785,0.125)$ & 0.168928 & 0.165777 & 0.165708 & 0.165508 \\
$(0.25,0.35)$ & 0.121888 & 0.121958 & 0.122014 & 0.121951 \\
$(0.465,0.175)$ & 0.141372 & 0.140138 & 0.140160 & 0.139990 \\
$(0.315,0.645)$ & 0.127239 & 0.127547 & 0.127580 & 0.127484 \\
$(0.1,0.2)$ & 0.108818 & 0.108820 & 0.108898 & 0.108911 \\
$(0.8,0.9)$ & 0.179177 & 0.166929 & 0.166861 & 0.166667 \\
\hline
\end{tabular}

$$
\bar{\lambda}_{i j}^{(0)}=\left[\begin{array}{ll}
1 & 0 \\
0 & 2
\end{array}\right], \quad \bar{\lambda}_{i j}^{(1)}=\left(2 \bar{x}_{1}+0.1 \bar{x}_{1}^{2}\right)\left[\begin{array}{ll}
1 & 0 \\
0 & 2
\end{array}\right] .
$$

For this problem an approximate solution is sought in the form (16) with one iteration; that is $\bar{T}=\bar{T}^{(0)}+\epsilon \bar{T}^{(1)}$, where $\bar{T}^{(0)}$ and $\bar{T}^{(1)}$ satisfy (10) and (17) for $r=0,1$ respectively. Table 1 compares the analytic and boundary element method (BEM) results for a number of points in the domain $\Omega$ and for the cases when the boundary $\partial \Omega$ is divided into 8,32 and 64 segments. For up to 32 boundary segments the accuracy of the solution improves as the number of segments increases. The error is then influenced by the truncation error due to the solution (16) being restricted to two terms. Thus any further increase in the number of boundary segments does not uniformly improve the accuracy of the results.

2. The coefficients (22) may also be written in the form

$$
\bar{\lambda}_{i j}=\left(1+0.1 \bar{x}_{1}\right)^{2}\left[\begin{array}{ll}
1 & 0 \\
0 & 2
\end{array}\right]
$$

by which way of presentation the coefficients also take form (2) 
TABLE 2: Numerical and analytic solutions for Problem 1

$\begin{array}{lllll}\text { Position } & \bar{T} \text { BEM } & \bar{T} \text { BEM } & \bar{T} \text { BEM } & \bar{T}\end{array}$

\begin{tabular}{ccccc}
$\left(\bar{x}_{1}, \bar{x}_{2}\right)$ & 8 segments & 32 segments & 64 segments & Analytic \\
\hline$(0.785,0.125)$ & 0.164404 & 0.165623 & 0.165532 & 0.165508 \\
$(0.25,0.35)$ & 0.121393 & 0.121860 & 0.121914 & 0.121951 \\
$(0.465,0.175)$ & 0.139896 & 0.139942 & 0.139968 & 0.139990 \\
$(0.315,0.645)$ & 0.126828 & 0.127403 & 0.127450 & 0.127484 \\
$(0.1,0.2)$ & 0.108679 & 0.108776 & 0.108859 & 0.108911 \\
$(0.8,0.9)$ & 0.169978 & 0.166808 & 0.166696 & 0.166667 \\
\hline
\end{tabular}

with

$$
g(\mathbf{x})=\left(1+0.1 \bar{x}_{1}\right)^{2}, \quad \bar{\lambda}_{i j}^{(0)}=\left[\begin{array}{ll}
1 & 0 \\
0 & 2
\end{array}\right], \quad \bar{\lambda}_{i j}^{(1)}=\left[\begin{array}{ll}
0 & 0 \\
0 & 0
\end{array}\right] .
$$

With this representation the integral equation (18) needs to be solved only for the first term (i.e. for $r=0$ ) of the series solution (16). The domain integral involved in the integral equation (18) is zero as the function $h^{(r)}=0$ for $r=0,1,2, \ldots$ and as a consequence $T^{(r)}=0$ for $r=1,2, \ldots$.

Table 2 shows a comparison between the computational results obtained using the BEM and analytic results for the same interior points. Only line integrals are involved in the BEM and the procedure used to evaluate these integrals is identical to that used for Case 1. The results displayed in Table 2 (which may be compared with the results in Table 1) improve in accuracy as the number of boundary segments increases. In particular for 64 segments the results in Table 2 are considerably better than the comparable results in Table 1. This is to be expected since the numerical method only involves boundary integrals and the error incurred in the previous case by truncating the solution (16) is not a feature of the method of this section. 


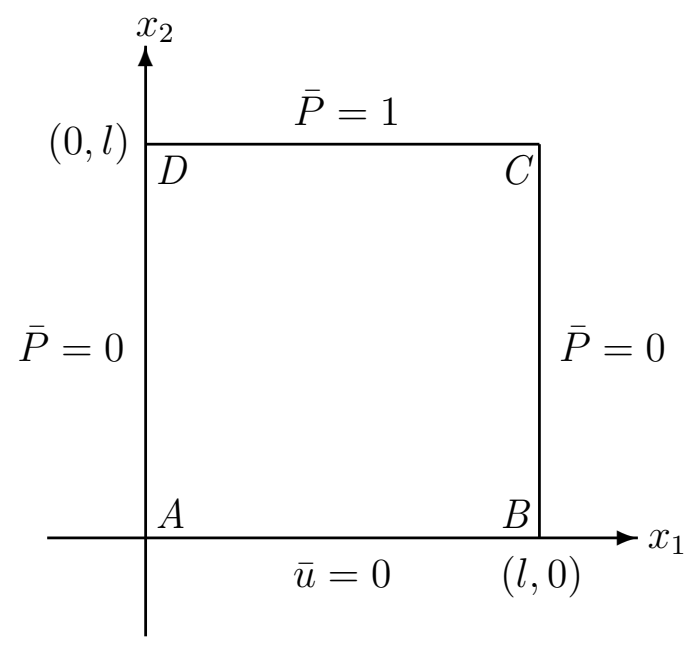

Figure 2: The geometry for Problem 2

\subsection{Problem 2}

Consider the elastostatic problem of determining the antiplane displacement $u$ for an inhomogeneous material occupying the square region shown in Figure 2 with the boundary conditions (as shown in Figure 2)

$$
\begin{aligned}
\bar{u} & =0 & & \text { on } \mathrm{AB}, \\
\bar{P} & =1 & & \text { on } \mathrm{CD}, \\
\bar{P} & =0 & & \text { on } \mathrm{BC} \text { and } \mathrm{AD},
\end{aligned}
$$

where $\bar{u}=u / u^{*}$ with $u^{*}$ a reference displacement and $\bar{P}=P / P^{*}$ where $P=\lambda_{i j}\left(\partial u / \partial x_{j}\right) n_{i}$ denotes the antiplane component of the stress vector and $P^{*}$ is a reference value of this component. The elastic moduli $\lambda_{i j}$ of the material increases in magnitude from $\bar{x}_{2}=0$ 
TABle 3: Numerical solutions for Problem 2 when $\beta=0$

\begin{tabular}{cccc}
$\begin{array}{c}\text { Position } \\
\left(\bar{x}_{1}, \bar{x}_{2}\right)\end{array}$ & $\begin{array}{c}\bar{u} \text { BEM } \\
\text { 160 segments }\end{array}$ & $\begin{array}{c}\bar{u} \text { BEM } \\
\text { 320 segments }\end{array}$ & $\begin{array}{c}\bar{u} \\
\text { Analytic }\end{array}$ \\
\hline$(0.5,0.1)$ & 0.0410 & 0.0413 & 0.0416 \\
$(0.5,0.3)$ & 0.0928 & 0.0932 & 0.0937 \\
$(0.5,0.5)$ & 0.1239 & 0.1244 & 0.1250 \\
$(0.5,0.7)$ & 0.1446 & 0.1452 & 0.1458 \\
$(0.5,0.9)$ & 0.1594 & 0.1600 & 0.1607 \\
\hline
\end{tabular}

to $\bar{x}_{2}=1$ according to the relationship

$$
\bar{\lambda}_{i j}=\left\{\left(1+2 \bar{x}_{2}\right)^{2}+0.1 \beta \sin \pi \bar{x}_{2}\right\}\left[\begin{array}{ll}
1 & 0 \\
0 & 2
\end{array}\right]
$$

where $\bar{\lambda}_{i j}=\lambda_{i j} / \lambda^{*}$ where $\lambda^{*}$ is a reference value of the elastic modulus. These moduli may be written in the form (2) with

$$
\begin{gathered}
g(\mathbf{x})=\left(1+2 \bar{x}_{2}\right)^{2}, \quad \epsilon=0.1 \\
\bar{\lambda}_{i j}^{(0)}=\left[\begin{array}{ll}
1 & 0 \\
0 & 2
\end{array}\right], \quad \bar{\lambda}_{i j}^{(1)}=\beta \sin \pi \bar{x}_{2}\left[\begin{array}{ll}
1 & 0 \\
0 & 2
\end{array}\right] .
\end{gathered}
$$

where $\bar{\lambda}_{i j}^{(0)}=\lambda_{i j}^{(0)} / \lambda$ and $\bar{\lambda}_{i j}^{(1)}=\lambda_{i j}^{(1)} / \lambda$.

For $\beta=0$ the problem has the analytic solution $\bar{u}=\bar{x}_{2} /[2(1+$ $\left.\left.2 \bar{x}_{2}\right)\right]$. Table 3 compares numerical values for this problem, obtained using the method of Section 3, and the corresponding values obtained from the analytic solution.

When $\beta=1$, there is no available exact analytic solution to this particular boundary value problem. Some numerical values obtained using the BEM of Section 3 are given in Table 4 . The values given indicate the expected convergence properties as the number of boundary segments increases. Also they are slightly smaller than 
TABLe 4: Numerical solutions for Problem 2 when $\beta=1$

\begin{tabular}{cccc}
$\begin{array}{c}\text { Position } \\
\left(\bar{x}_{1}, \bar{x}_{2}\right)\end{array}$ & $\begin{array}{c}\bar{u} \text { BEM } \\
80 \text { segments }\end{array}$ & $\begin{array}{c}\bar{u} \text { BEM } \\
160 \text { segments }\end{array}$ & $\begin{array}{c}\bar{u} \text { BEM } \\
320 \text { segments }\end{array}$ \\
\hline$(0.5,0.1)$ & 0.0385 & 0.0392 & 0.0395 \\
$(0.5,0.3)$ & 0.0888 & 0.0897 & 0.0901 \\
$(0.5,0.5)$ & 0.1192 & 0.1201 & 0.1206 \\
$(0.5,0.7)$ & 0.1394 & 0.1404 & 0.1410 \\
$(0.5,0.9)$ & 0.1540 & 0.1550 & 0.1556 \\
\hline
\end{tabular}

the corresponding values given in Table 3 for the case when $\beta=0$. This is to be expected on physical grounds since the rigidity of the material as reflected in the larger value of the coefficients $\bar{\lambda}_{i j}$ when $\beta=1$ would give rise to smaller values of the displacement.

\section{Summary}

A boundary element method for the solution of certain classes of elliptic boundary value problems for anisotropic inhomogeneous media has been derived. The method is generally easy to implement to obtain numerical values for particular problems. It can be applied to a wide class of practical problems for inhomogeneous anisotropic and functionally graded materials and the analysis includes isotropy as a special case. The numerical results obtained using the method indicate that it can provide accurate numerical solutions to particular boundary value problems. 


\section{References}

[1] W. T. Ang, J. Kusuma and D. L. Clements. A boundary element method for a second order elliptic partial differential equation with variable coefficients. Engng. Anal. Boundary Elements, 18:311-316, 1997. C80

[2] A. H.-D. Cheng. Darcy's Flow with Variable Permeability: A Boundary Integral Solution. Water Resources Research, 20:980-984, 1984. C80

[3] A. H.-D. Cheng. Heterogeneities in flows through porous media by boundary element method. Topics in Boundary Element Research: Applications to Geomechanics, 4:1291-1344, 1987. C80

[4] D. L. Clements A boundary integral equation method for the numerical solution of a second order elliptic equation with variable coefficients. J. Austrl. Math. Soc, 22(B):218-228, 1980. C80

[5] D. L. Clements. Boundary Value Problems Governed by Second Order Elliptic Systems. Pitman, 1981. C84, C87

[6] D. L. Clements and W. S. Budhi. A Boundary Element Method for the Solution of A Class of Steady-State Problems for Anisotropic Media. Journal of Heat Transfer, 121, 1999. C81

[7] D. L. Clements and C. Rogers. Wave propagation in inhomogeneous elastic media with $(\mathrm{N}+1)$-dimensional spherical symmetry. Canadian J. Phys., 52:1246-1252, 1974. C80 
[8] G. S. Gipson, J. C. Ortiz and R. P. Shaw. Two-dimensional linearly layered potential flow by boundary elements. Boundary Elements XVII, C. A. Brebbia ed. Springer-Verlag, Berlin, 1995. C80

[9] R. Rangogni. A solution of Darcy's flow with variable permeability by means of B.E.M and perturbation techniques. Boundary Elements IX, Vol. 3, C. A. Brebbia ed. Springer-Verlag, Berlin, 1987. C80

[10] R. P. Shaw. Green's functions for heterogeneous media potential problems. Engng. Anal. Boundary Elements, 13:219-221, 1994. C80 